\title{
Parasitism potential of Dirhinus giffardii (Silvestri) (Hymenoptera: Chalcididae) on pupae of the fruit fly species, Zeugodacus cucurbitae (Coquillett) and Bactrocera dorsalis (Hendel) (Diptera: Tephritidae), during variable exposure durations
}

Fazl Ullah, Muzammil Farooq* (D), Sabyan Faris Honey and Naeem Zada

\begin{abstract}
Background: Fruit flies are important insect pests of horticultural crops. Pesticides used to control them which cause environmental and health hazards; therefore, other alternative sustainable management measures are required.

Main body: Successful implementation of an integrated pest management program, using biological control agents, needs synchronization of parasitoids with hosts selection and exposure time. The present study was conducted to assess the biological activity of the parasitoid species, Dirhinus giffardii (Silvestri) (Hymenoptera: Chalcididae), against the melon fruit fly, Zeugodacus cucurbitae (Coquillett), and the oriental fruit fly, Bactrocera dorsalis (Hendel) (Diptera: Tephritidae), at different exposure durations (24, 48, and $72 \mathrm{~h}$ ). The experiments were conducted under standard laboratory conditions. Significant differences in the parasitism rate of D. giffardii were observed for both species at different exposure durations. The maximum parasitism rate of D. giffardii (52.60 \pm $2.84 \%)$ and $(42.73 \pm 2.74 \%)$ was observed at 48 -h exposure period for $Z$. cucurbitae and B. dorsalis, respectively. Also, a comparison between both species showed a difference in parasitism rate at 24 and $48 \mathrm{~h}$ and a positive correlation between percent parasitism and exposure duration. Adult emergence of D. giffardii showed a maximum emergence rate from pupae of $Z$. cucurbitae $(63.55 \pm 4.02 \%)$ and B. dorsalis $(51.61 \pm 2.33 \%)$ at 10 days interval, respectively. However, the daily emergence of the parasitoid was not correlated to exposure duration in both species. The results of the present study may serve in the mass rearing of D. giffardii.

Conclusion: The biological activity of D. giffardii was maximum at $48 \mathrm{~h}$ of exposure for both fruit fly species, and this important information may improve the mass rearing technology of D. giffardii.
\end{abstract}

Keywords: Dirhinus giffardii, Bactrocera dorsalis, Zeugodacus cucurbitae, Fruit flies, Exposure time

\footnotetext{
* Correspondence: m.faroog@cabi.org

Centre for Agriculture and Bioscience International (CABI), Regional

Bioscience Centre, 1-A Opposite Data Gunj Bakhsh Road, P.O Box \# 8,

Rawalpindi, Pakistan
}

\section{Springer Open}

(c) The Author(s). 2021 Open Access This article is licensed under a Creative Commons Attribution 4.0 International License, which permits use, sharing, adaptation, distribution and reproduction in any medium or format, as long as you give appropriate credit to the original author(s) and the source, provide a link to the Creative Commons licence, and indicate if changes were made. The images or other third party material in this article are included in the article's Creative Commons licence, unless indicated otherwise in a credit line to the material. If material is not included in the article's Creative Commons licence and your intended use is not permitted by statutory regulation or exceeds the permitted use, you will need to obtain permission directly from the copyright holder. To view a copy of this licence, visit http://creativecommons.org/licenses/by/4.0/. 


\section{Background}

Fruit flies are positioned as quarantine pests of horticultural crops worldwide (Mariana et al. 2020). In South Asia, fruit fly species are reported for more than 100 host plants (Hui and Liu 2005). In Pakistan, losses of US\$ 200 million annually are reported due to fruit fly species mainly the oriental fruit fly, Bactrocera dorsalis (Hendel) (Diptera: Tephritidae), and the melon fruit fly, Bactrocera (Zeugodacus) cucurbitae (Coquillett) (Diptera: Tephritidae) (Sarwar 2014). Most of the available literatures use Bactrocera (Zeugodacus) cucurbitae as a scientific name. However, considering the revised taxonomic position for the B. cucurbitae as proposed by Virgilio et al. (2015), this manuscript will consider Zeugodacus cucurbitae as a scientific name for the melon fly.

Currently, chemical sprays and pheromone traps are considered the first line of defense against fruit fly infestation. However, lack of awareness and injudicious use of insecticides caused many problems including reduced chemical efficacy, resistance in fruit fly, and residual effects (Aziz and Hussain 2018). These mentioned problems triggered the efforts to create awareness among the farming community and use other alternatives ecofriendly and sustainable management techniques (Vargas et al. 2012). In this regard, Centre for Agriculture and Bioscience International (CABI) through its regional bioscience center in Pakistan implemented a USAID/USDA funded project entitled "Phytosanitary Risk Management Programme in Pakistan (PRMP)" in 2014-2019. The main objective of this program was to explore and utilize the local species of biocontrol agents for the management of apple codling moth, papaya mealy bug, and fruit flies.

For the fruit fly management, the extensive surveys were conducted in different fruit and vegetable growing areas of Pakistan, which resulted in the exploration of Dirhinus giffardii (Silvestri) (Hymenoptera: Chalcididae) associated with fruit fly species as a pupal parasitoid. In the past, D. giffardii initially described from Nigeria has been considered as a generalist parasitoid for integrated pest management programs. It attacks several families, i.e., Tephritidae, Glossinidae, and Muscidae of order Diptera and Noctuidae of order Lepidoptera (Noyes 2014). In the management of fruit flies, D. giffardii showed good parasitism rates on pupae of different fruit fly species, i.e., $B$. dorsalis, $B$. cucurbitae, $B$. zonata, $B$. oleae, and B. correcta (Naveed et al. 2014; Rasool et al. 2017). Dirhinus giffardii completes a major part of its life cycle (egg to pupa) inside the host pupae. The parasitoid consumes the host resource completely and gained more fitness when reared on a large host (Imran et al. 2013). However, utilizing biological control agents in pest management programs requires successful mass rearing techniques. Mass rearing optimization, synchronization of D. giffardii with host selection, and exposure time are key factors (Montoya et al. 2007).

In this regard, the present study was designed to determine suitable host and time required for maximum parasitism and for developing mass rearing protocol for D. giffardii.

\section{Main text \\ Materials and methods \\ Fruit fly cultures}

Parent colonies of the two fruit fly species, i.e., Z. cucurbitae and $B$. dorsalis, were maintained at CABI's Biological Control Laboratory, Rawalpindi, Pakistan (33" $38^{\prime} 39^{\prime} \mathrm{N}, 73^{\prime \prime} 04^{\prime} 57^{\prime}$ ), since 2016. The flies were kept in rearing cages $(22 \times 18 \times 18)$ (inches) equipped with iron mesh $(16 \mu \mathrm{m})$ to facilitate the ventilation and fine muslin cloth sleeve opening to provide the food for culture handling and maintenance. A standardized diet was provided for adult feeding (Table 1) pasted on small circular $(10 \mathrm{~cm}$ diameter) rexine sheets and replenished on a daily basis in rearing cages. Water was provided as ad libitum. The rearing conditions were maintained at $28 \pm$ $1{ }^{\circ} \mathrm{C}, 65 \pm 5 \% \mathrm{RH}$, and 14:10 (L to D) h photoperiod.

\section{Parasitoid's culture}

The parent colonies of $D$. giffardii were maintained since 2016. Initially, soil sieving was done for pupae collection from Sharaqpur district (31" 27'48' N 74" 6' $0^{\prime}$ E) Punjab, Pakistan. Later, sieved pupae were brought to the laboratory for further process. D. giffardii emerged from these pupae were kept in transparent cages for further propagation. An artificial diet containing solutions of $70 \%$ water and $30 \%$ honey was provided to adult parasitoid through soaked cotton on regular basis. The parent colony was reared at for 3 consecutive generations, and the rearing conditions were maintained at $28 \pm 1{ }^{\circ} \mathrm{C}$, $65 \pm 5 \% \mathrm{RH}$, and 14:10 (L to D) h photoperiod, before the experimentation to avoid pre-imaginal conditioning.

\section{Experimental units}

The host pupae of $Z$. cucurbitae and B. dorsalis (3 days old) were exposed to $D$. giffardii at different exposure durations, i.e., 24,48 , and $72 \mathrm{~h}$ (separated for each

Table 1 Composition of standardized diet for feeding the adults of fruit flies

\begin{tabular}{ll}
\hline Ingredients & Quantity (gm or $\mathbf{~ m l ) - f o r ~} \mathbf{8 0 0} \mathbf{~ m l ~ d i e t ~}$ \\
\hline Yeast & 03 \\
Banana & 1392 \\
Egg yolk & 06 \\
Vitamin B complex & 02 \\
Sugar & 33 \\
\hline
\end{tabular}


species). The experiment was conducted with 3 replicates for each exposure duration. Each replicate was 500 pupae (3 days old) of $Z$. cucurbitae and B. dorsalis (from each species), placed in a small Petri dish $(6 \mathrm{~cm}$ diameter $)$ as residing media for $D$. giffardii adults to parasitize them. Ten pairs of D. giffardii (5 days old) were introduced to individual replicates of $Z$. cucurbitae and B. dorsalis pupae separately and exposed for said exposure durations. Later, the exposed pupae were removed from the containers and kept in separate boxes to record the parasitism and emergence data for 15 consecutive days. The experiments were repeated twice in time and pooled together for precise results.

\section{Statistical analysis}

The parasitism rate was calculated using the following formula as per Farooq et al. (2019):

Parasitism rate $=$ number of parasitiods (emerged + unemerged $)$

$/$ total number of pupae $\times 100$

Experimental data was subjected to analysis of variance (ANOVA) in Statistix 8.1 (McGraw-Hill 2008), and multiple comparisons between means and groups were made with aid of LSD test at $0.05 \%$ level of significance. The linear regression was used to check the relationship between percent parasitism and percent emergence to varying exposure durations.

\section{Results and discussion}

\section{Parasitism rate}

The parasitism rate of $D$. giffardii after exposure to $Z$. cucurbitae pupae was determined. The results showed that the highest parasitism rate $(52.60 \pm 2.84 \%)$ by $D$. giffardii was observed after $48 \mathrm{~h}$ exposure to Z. cucurbitae pupae, followed by $72 \mathrm{~h}$ exposure $(37.73 \pm 4.80 \%)(F=$ 8.67; $\mathrm{df}=2,8 ; P=0.017$ ) (Fig. 1). For $B$. dorsalis, the highest parasitism rate $(42.73 \pm 2.74 \%)$ by $D$. giffardii was observed after $48 \mathrm{~h}$ exposure to $B$. dorsalis pupae, followed by $72 \mathrm{~h}$ exposure $(39.47 \pm 1.39 \%)(F=20.1$; df $=2,8 ; P=0.002$ ) (Fig. 1). In addition, the comparison between parasitism rate for different pupae groups and different exposure durations showed significant difference at 24 and $48 \mathrm{~h}(F=4.75 ; \mathrm{df}=2,8 ; P=0.03)$ (Fig. 1). In the context of correlation, the results showed a positive correlation for parasitism rate of $D$. giffardii vs. exposure duration for $Z$. cucurbitae pupae $\left(R^{2}=\right.$ 0.7172) (Fig. 2a) and B. dorsalis pupae $\left(R^{2}=0.9181\right)$ (Fig. 2b).

Parasitoids can discriminate between host pupae. They provide a survival advantage to parasitoids population and the preference of $D$. giffardii that may be influenced by different hosts as they possess different nutritional qualities (Harvey 2000). The present study highlighted on the parasitism activity of $D$. giffardii on $B$. dorsalis and $Z$. cucurbitae. The parasitism potential of D. giffardii against $B$. dorsalis and $Z$. cucurbitae might be a virtuous source for controlling fruit flies (Van Lenteren et al. 2006). The results of the present study showed significant differences in parasitism rates at different exposure

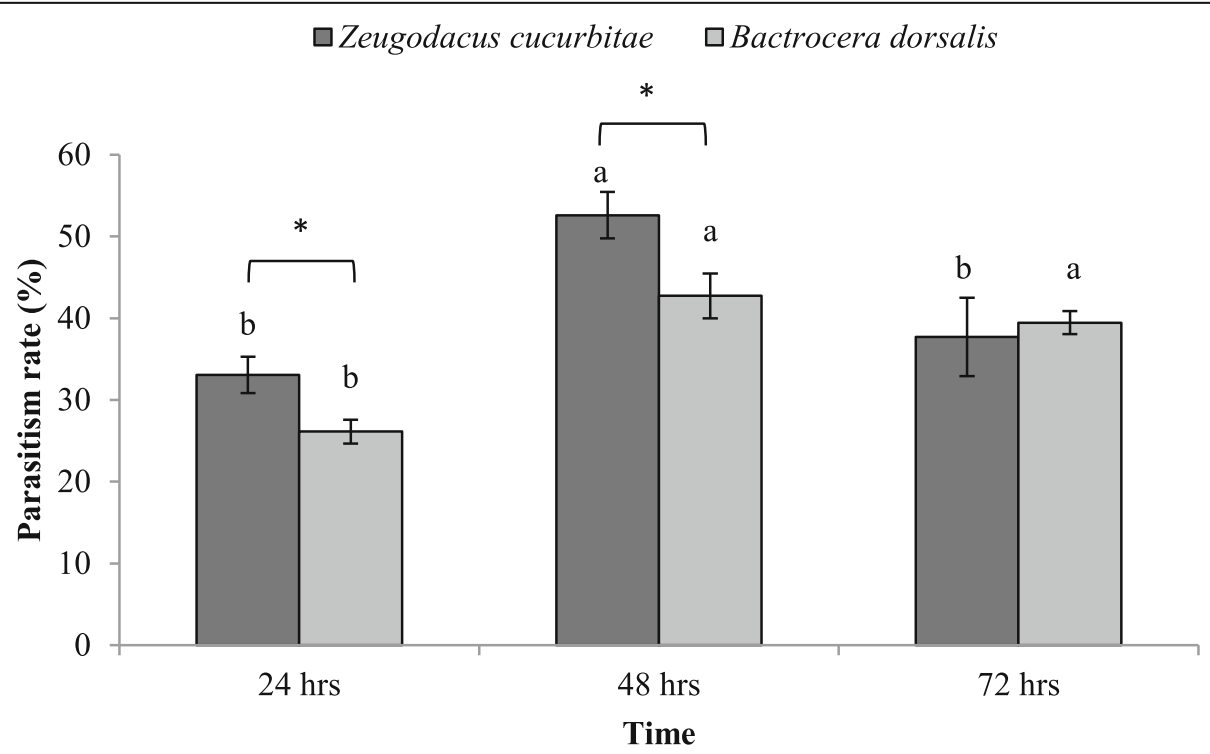

Fig. 1 Parasitism rate of Dirhinus giffardii after 24, 48, and $72 \mathrm{~h}$ exposures subjected for Z. cucurbitae and B. dorsalis pupae. Means followed by similar letter are non-significantly different at $0.05 \%$ level of significance (for black and white columns), while ns present non-significant difference at $0.05 \%$ level of significance. Asterisk $\left(^{*}\right)$ shows significant difference between the treatments and different pupae groups 

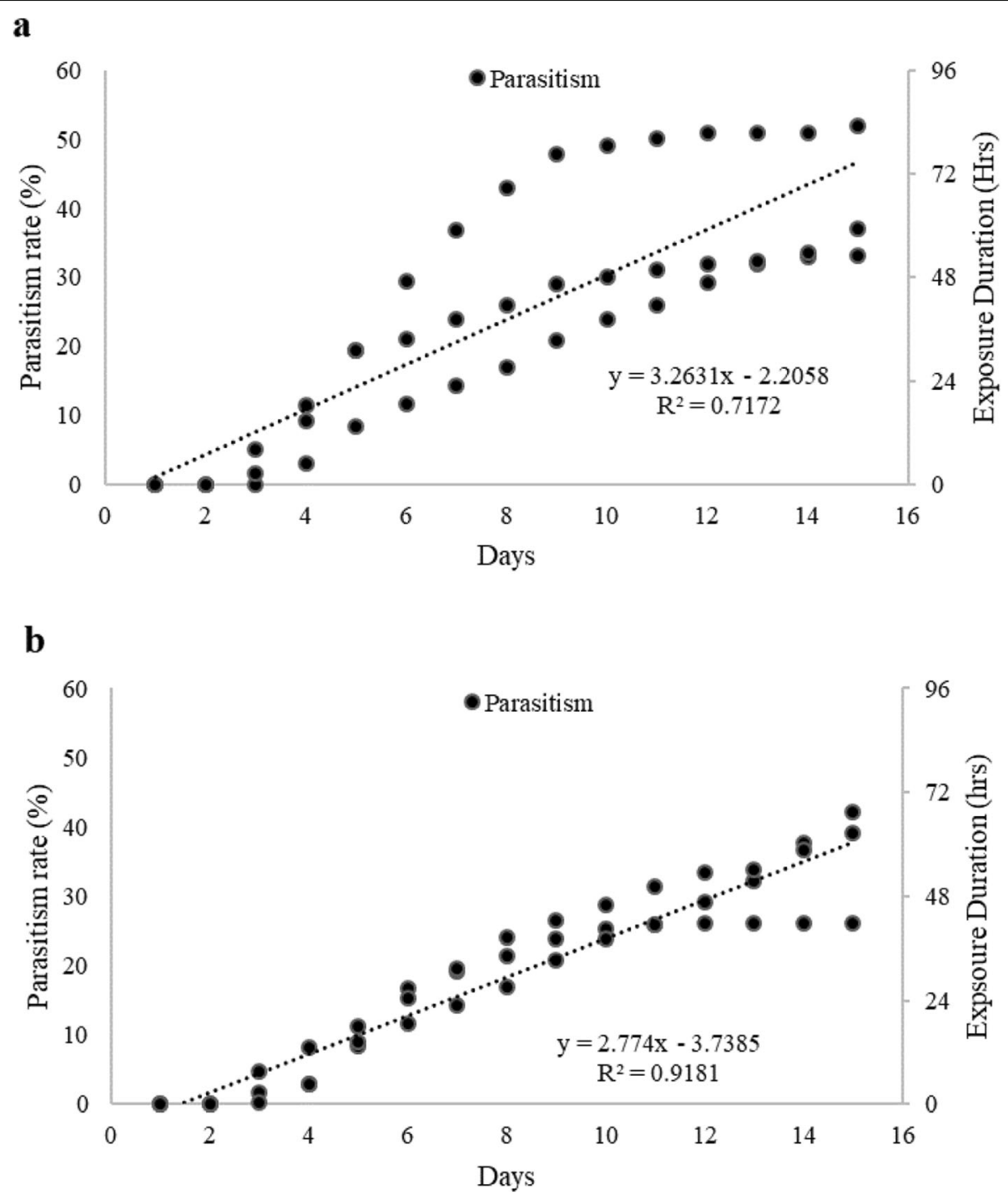

Fig. 2 Correlation between parasitism rate of Dirhinus giffardii and exposure duration a Zeugodacus cucurbitae and $\mathbf{b}$ Bactrocera dorsalis pupae

durations for both species of fruit flies. The maximum emergence rate of $D$. giffardii was observed on Z. cucurbitae than that on $B$. dorsalis pupae. Similar results were observed by Rasool et al. (2017), where Z . cucurbitae pupae showed a maximum parasitism rate of $D$. giffardii. The present study focused mainly on exposure duration and its effect on the parasitism activity of $D$. giffardii. The parasitoid showed the maximum parasitism rate of $Z$. cucurbitae and B. dorsalis pupae after $48 \mathrm{~h}$ of exposure. Kacar et al. (2017) described the parasitoids functional response in which the maximum number of parasitoids was observed after the hosts pupae were exposed for $48 \mathrm{~h}$ to parasitoids which support findings of the current study.

\section{Adult emergence}

Adult emergence data of $D$. giffardii from $Z$. cucurbitae pupae was determined at 5-, 10-, and 15-day intervals.
The maximum adult emergence of $D$. giffardii $(63.55 \pm$ 4.02) was observed on 10-day intervals after exposure of $24 \mathrm{~h}$. The minimum adult emergence $(13.85 \pm 4.20)$ was observed at 5-day intervals after exposure of $72 \mathrm{~h}(F=$ 2.78; $\mathrm{df}=4,26 ; P=0.0473$ ) (Fig. 3).

Additionally, adult emergence data of $D$. giffardii for B. dorsalis pupae was also determined at 5-, 10-, and 15-day intervals. The maximum emergence rate of D. giffardii $(51.67 \pm 2.33 \%)$ was recorded at the 10-day intervals after exposure of $24 \mathrm{~h}$. Minimum emergence $(11.66 \pm 0.50 \%)$ was observed at 15 -day intervals after exposure of $72 \mathrm{~h}(F=4.61 ; \mathrm{df}=4,26 ; P$ $=0.006$ ) (Fig. 3).

In the context of correlation, the results showed no correlation for the daily emergence of $D$. giffardii vs. exposure duration for $Z$. cucurbitae pupae $\left(R^{2}=0.0085\right)$ (Fig. 4a) and B. dorsalis pupae $\left(R^{2}=0.004\right)$ (Fig. 4b). 


\section{$\square$ Zeugodacus cucurbitae $\square$ Bactrocera dorsalis}

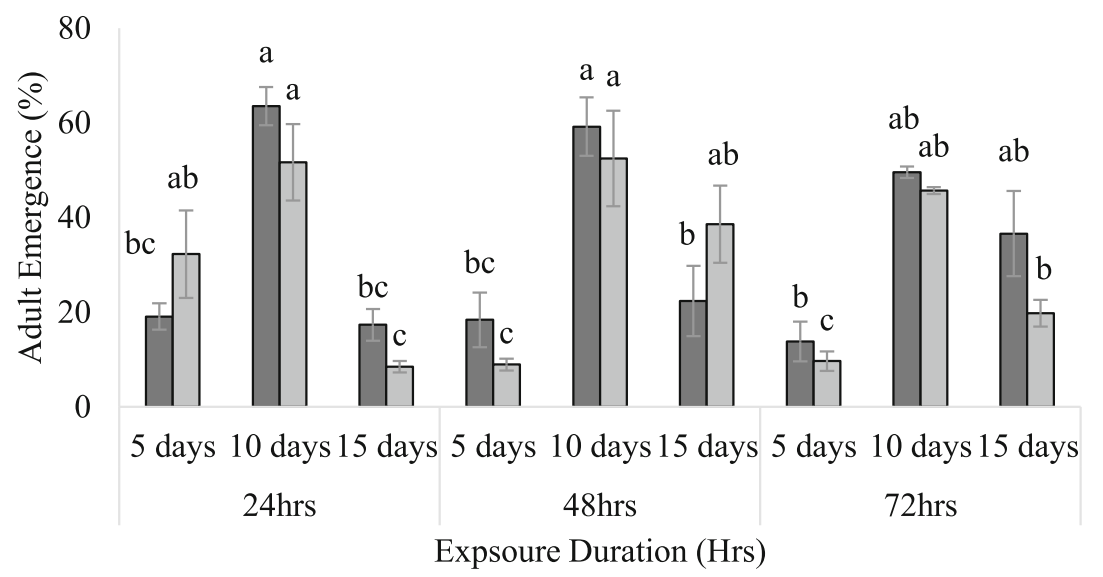

Fig. 3 Adult emergence of Dirhinus giffardii after 24, 48, and $72 \mathrm{~h}$ exposures subjected for Zeugodacus cucurbitae pupa and Bactrocera dorsalis pupae. Means followed by similar letter are not significantly different at $0.05 \%$ level of significance (for white and black columns)
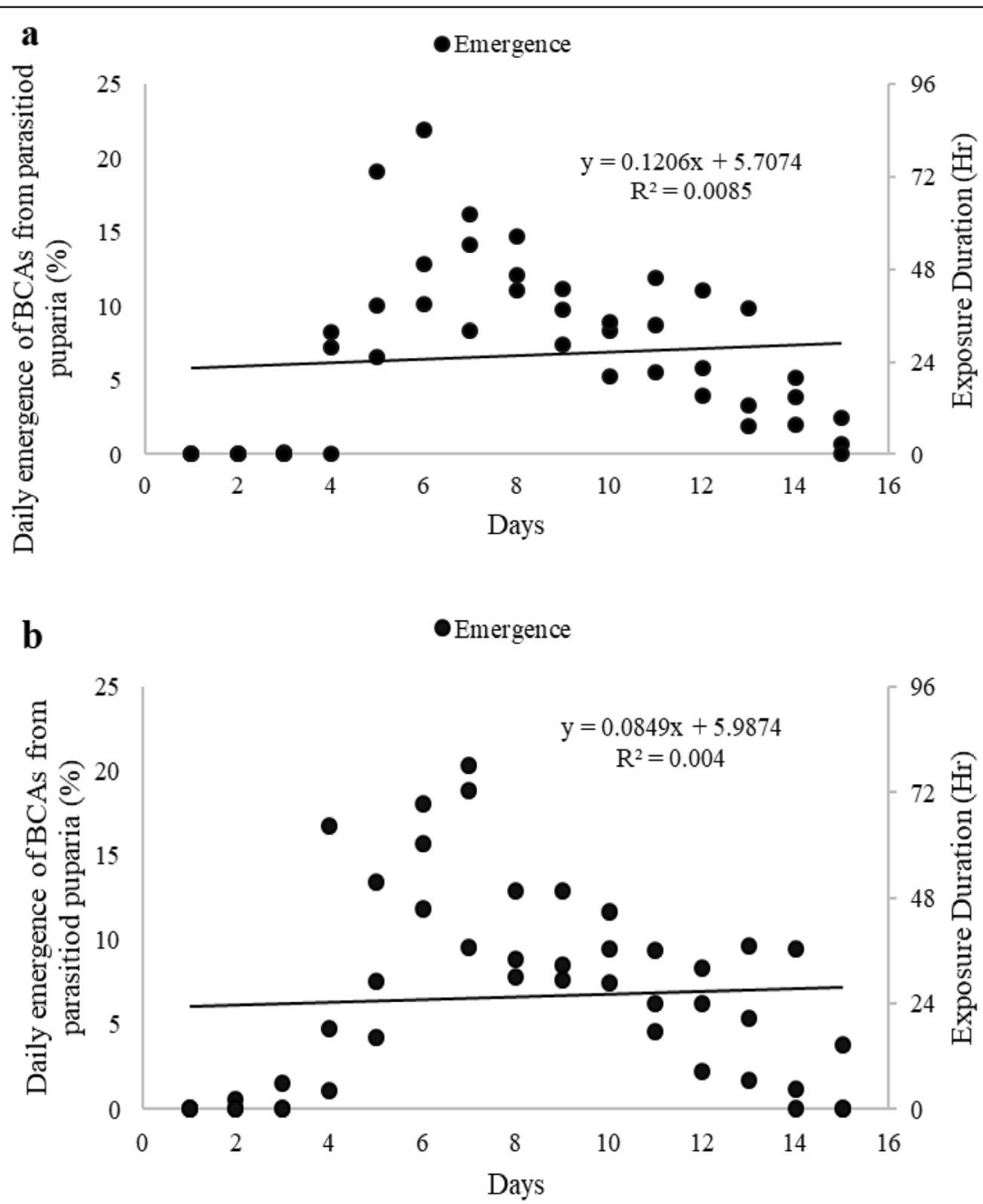

Fig. 4 Correlation between adult emergence of Dirhinus giffardii and exposure duration a Zeugodacus cucurbitae and $\mathbf{b}$ Bactrocera dorsalis pupae 
The present study demonstrated the adult emergence of the parasitoids from infested hosts in relation to days. Interestingly, in the case of $D$. giffardii and $B$. dorsalis, a significant difference was observed for different exposure times; however, maximum emergence occurred at 10day intervals for all exposure durations. Similarly, the results were observed for the D. giffardii and Z. cucurbitae, where a maximum emergence of flies and parasitoids occurred at 10-day intervals, although the present study showed that exposure duration had a significant effect on the adult emergence of parasitoids. However, Guillen et al. (2002) reported non-significant differences in parasitism rates of Coptera haywardi (Hymenoptera: Diapriidae) and Pachycrepoideus vindemiae Rondani (Hymenoptera: Pteromalidae) after exposure periods of 24, 36, 48, and $72 \mathrm{~h}$.

Besides, the age of host, food source, and age of parasitoids are important factors that play a vital role in the development of parasitoids (Sitthichaiyakul and Amornsak 2017; Naveed et al. 2014). Keeping in view the importance of biological control and potential of $D$. giffardii, future studies will be conducted aiming on the assessment of parasitism and host preference of D. giffardii under choice and non-choice scenarios. Also, the present studies were conducted in the laboratory conditions and small arenas, which may not reflect field conditions. However, choose the scenario that could help us to identify some key factors, i.e., host selection and exposure time may aid in improving mass rearing protocols and comply with demands for rearing and maintenance within the context of research or commercialization.

\section{Conclusion}

In conclusion, the current study was performed to assess the biological activity of D. giffardii for fruit flies. D. giffardii emergence was maximum at $48 \mathrm{~h}$ of exposure duration for Z. cucurbitae $(52.60 \pm 2.84 \%)$ and B. dorsalis $(42.73 \pm 2.74 \%)$. Further, the results showed a correlation between parasitism to increasing exposure duration. The current study provides important information for mass rearing of $D$. giffardii, as comparing relative effectiveness and exposure duration of $D$. giffardii for fruit fly species could aid in improve rearing technology of this biocontrol agent.

\section{Abbreviations}

ANOVA: Analysis of variance; LSD: Least significance difference

\section{Acknowledgements}

We thank Mr. Riaz Mahmood for providing technical inputs in this study. Acknowledgement is also made to USAID/USDA for providing financial support through the project "Phytosanitary Risk Management Program in Pakistan" to conduct this study.

\section{Authors' contributions}

The concept and design of experiments were prepared by all authors. FA and $\mathrm{NZ}$ conducted the experiments in the laboratory. MF, SH, and FA wrote the manuscript and analyzed the data. All authors read and approved the manuscript.

\section{Funding}

We gratefully acknowledge the funding provided for this research by United States Agency for International Development (USAID) through United States Department of Agriculture (USDA) under the CABI project "Phytosanitary Risk Management Program in Pakistan" (PRMP).

$C A B I$ is an international intergovernmental organization, and we gratefully acknowledge the core financial support from our member countries (and lead agencies) including the UK (Foreign, Commonwealth \& Development Office), China (Chinese Ministry of Agriculture), Australia (Australian Centre for International Agricultural Research), Canada (Agriculture and Agri-Food Canada), Netherlands (Directorate-General for International Cooperation), and Switzerland (Swiss Agency for Development and Cooperation). See https:// www.cabi.org/about-cabi/who-we-work-with/key-donors/ for full details.

\section{Availability of data and materials}

All data of the study have been presented in the manuscript, and highquality and grade materials were used in this study.

Ethics approval and consent to participate

Not applicable

\section{Consent for publication}

Not applicable

\section{Competing interests}

The author(s) have not declared any conflict of interest.

Received: 26 August 2020 Accepted: 17 December 2020

Published online: 05 January 2021

\section{References}

Aziz SS, Hussain M (2018) Fruit pest management in Pakistan: a review. Sci Inq Rev 2:43-52

Farooq M, Fazalullah, Sangita B, lqbal H, Sabyan FH (2019) In vitro host preference and influence of parasitoids-host density on Dirhinus giffardii for Bactrocera cucurbitae and Bactrocera dorsalis pupae. Acad J Agric Res 7:224-231

Guillen L, Aluja M, Equihua M, Sivinski I (2002) Performance of two fruit fly (Diptera: Tephritidae) pupal parasitoids (Coptera haywardi [Hymenoptera: Diapriidae] and Pachycrepoideus vindemiae [Hymenoptera: Pteromalidae]) under different environmental soil conditions. Biol Control 23:219-227

Harvey JA (2000) Dynamic effects of parasitism by an endoparasitoid Wasp on the development of two host species: implications for host quality and parasitoid fitness. Ecol Entomol 25:267-278

Hui Y, Liu J (2005) Population dynamics of the oriental fruit fly, Bactrocera dorsalis (Diptera: Tephritidae) in the Kunming area, southwestern China. Insect Sci 12:387-392

Imran R, Ahmad N, Rashdi SMMS, Ismail M, Khan MH (2013) Laboratory studies on ovipositional preference of the peach fruit fly Bactrocera zonata (Saunders) (Diptera: Tephritidae) for different host fruits. Afr J Agric 8:1300-1303

Kacar G, Wang XG, Biondi A, Daane KM (2017) Linear functional response by two pupal Drosophila parasitoids foraging within single or multiple patch environments. PLOS ONE 12(8):e0183525

Mariana M, Humberto MM, Silvia B, Claudia C, Karina G, Brenda M, Jorge T, Pablo L, Elias D, Vangelis D, Georgios A, Nikos T, Antonios A, Diego F, George T (2020) Wolbachia pipientis associated with tephritid fruit fly pests: from basic research to applications. Front Microbiol 11:1-23. https://doi.org/10.3389/ fmicb.2020.01080

McGraw-Hill C (2008) Statistix 8.1 analytical software. Tallahassee

Montoya P, Cancino J, Zenil M, Santiago G, Gutierrez JM (2007) The augmentative biological control component in the Mexican National Campaign against Anastrepha spp. fruit flies. In: Vreysen MJB, Robinson AS, Hendrichs I (eds) Area-wide control of insects pests: from research to field implementation. Springer, Dordrecht, pp 661-670 
Naveed M, Suhail A, Ahmad N, Rauf I, Akbar W (2014) Role of Dirhinus giffardii Silv. age on the parasitism preference to different days old pupae of Bactrocera zonata and Bactrocera cucurbitae. J Agric Biotechnol Sustain Dev 6:1-5

Noyes JS (2014) Universal Chalcidoidea data base. Available from: http://www. nhm.ac.uk/chalcidoids

Rasool B, Rafique M, Asrar M, Rasool R, Adeel M, Rasul A, Jabeen F (2017) Host preference of Bactrocera flies species (Diptera: Tephritidae) and parasitism potential of Dirhinus giffardii and Pachycropoideus vindemmiae under laboratory conditions. Pak Entomol 39:17-21

Sarwar M (2014) Some insect pests (Arthropoda: Insecta) of summer vegetables, their identification, occurrence, damage and adoption of management practices. Int I Sustain Agric Res 1:108-117

Sitthichaiyakul S, Amornsak W (2017) Host-substrate preference of Theocolax elegans (Westwood) (Hymenoptera: Pteromalidae), a larval parasitoid of the maize weevil, Sitophilus zeamais (Motschulsky) (Coleoptera: Curculionidae). Agric Nat Resour 51:36-39

Van Lenteren JC, Bale J, Bigler F, Hokkanen HMT, Loomans AJM (2006) Assessing risks of releasing exotic biological control agents of arthropod pests. Annu Rev Entomol 51:609-634

Vargas RI, Leblanc L, Harris EJ, Manoukis NC (2012) Regional suppression of Bactrocera fruit flies (Diptera: Tephritidae) in the Pacific through biological control and prospects for future introductions into other areas of the world. Insects 3:727-742

Virgilio M, Jordaens K, Verwimp C, White IM, De Meyer M (2015) Higher phylogeny of frugivorous flies (Diptera, Tephritidae, Dacini): localised partition conflicts and a novel generic classification. Mol Phylogenet Evol 85: 171-179. https://doi.org/10.1016/j.ympev.2015.01.007

\section{Publisher's Note}

Springer Nature remains neutral with regard to jurisdictional claims in published maps and institutional affiliations.

\section{Submit your manuscript to a SpringerOpen ${ }^{\circ}$ journal and benefit from:}

- Convenient online submission

- Rigorous peer review

- Open access: articles freely available online

High visibility within the field

- Retaining the copyright to your article

Submit your next manuscript at $\boldsymbol{\nabla}$ springeropen.com 